DOI: $10.1002 /$ adfm.201702253

Article type: Full Paper

\title{
Universal Control on Pyro-resistive Behavior of Flexible Self-regulating Heating Devices
}

Yi Liu, Han Zhang*, Harshit Porwal, Wei Tu, Jamie Evans, Mark Newton, James Busfield, Ton Peijs, and Emiliano Bilotti*

Y. Liu, Dr. H. Zhang, Dr. H. Porwal, Prof. J.J.C. Busfield, Prof. T. Peijs, Dr. E. Bilotti School of Engineering and Materials Science, Queen Mary University of London, Mile End Road, London E1 4NS, UK

E-mail: han.zhang@qmul.ac.uk (H. Zhang); e.bilotti@qmul.ac.uk (E. Bilotti)

J. Evans, M. Newton

LMK Thermosafe Ltd., 9-10 Moonhall Business Park, Helions Bumpstead Rd, Haverhill, Suffolk CB9 7AA, UK

Dr. H. Zhang, Dr. H. Porwal, Dr. W. Tu, Prof. T. Peijs, Dr. E. Bilotti

Nanoforce Technology Ltd., Joseph Priestley Building, Queen Mary University of London, Mile End Road, E1 4NS London, UK

Keywords: conductive polymer composites, positive temperature coefficient, smart heaters, self-regulating, Carbon nanotubes

\begin{abstract}
Smart heating devices with reliable self-regulating performances and high efficiency, combined with additional properties like mechanical flexibility, are of particular interest for applications in healthcare, soft robotics and smart building environments. Unfortunately, the development of smart heaters necessitates managing normally conflicting requirements such as good self-regulating capabilities and efficient Joule heating performances. Here, a simple and universal materials design strategy, based on a series connection of different conductive polymer composites (CPCs), is shown to provide unique control over the pyro-resistive properties. Hooke's and Kirchhoff's laws of electrical circuits can simply predict the overall pyro-resistive behavior of devices connected in either series or parallel configurations, hence providing design guidelines. An efficient and mechanically flexible Joule heating device is hence designed and created. The heater is characterized by a zero temperature coefficient of
\end{abstract}




\section{WILEY-VCH}

resistance below the self-regulating temperature, immediately followed by a large and sharp positive temperature coefficient (PTC) behavior with a PTC intensity of around $10^{6}$. Flexibility and toughness was provided by the selected elastomeric thermoplastic polyurethane (TPU) matrix as well as the device design. The universality of the approach is demonstrated by using different polymer matrices (TPU and HDPE) and conductive fillers (silver coated glass spheres $(\mathrm{AgS})$, carbon nanotubes (CNT) and graphite nanoplatelets (GNP)), for which repeatable results are consistently obtained. (Protected by International Patent Pending)

\section{Introduction}

Conductive polymer composites (CPCs) are considered as smart materials which have attracted significant interest from both academia and industry for decades as they combine the merits of plastics and conductive fillers. ${ }^{[1]}$ Their capability of detecting and responding to external stimuli has offered a range of promising applications by performing both sensing (damage, ${ }^{[2]}$ strain,${ }^{[3]}$ humidity, ${ }^{[4]}$ vapor, ${ }^{[5]}$ degradation, ${ }^{[6]}$ and current-limiting ${ }^{[7]}$ ) and actuating (artificial muscles ${ }^{[8]}$ and electroactive shape-memory ${ }^{[9]}$ ) functions. In particular, CPCs are practical choices for pyro-resistive applications like self-regulating heaters, temperature sensors and safe battery switches, due to their capability of changing electrical resistivity upon heating. ${ }^{[10]}$

Since their introduction in the late 1970s, self-regulating heating devices have been widely adopted in applications like domestic heaters and de-icing units. These smart heaters can operate at a nearly constant temperature over a broad range of voltage and dissipative conditions by utilizing the positive temperature coefficient (PTC) effect. The PTC effect, where the electrical resistivity increases with increasing operating temperature, is the main property that provides the fundamental function of self-regulating heating devices. ${ }^{[1]}$ The PTC phenomenon is generally explained by the mismatch of thermal expansion between the polymer matrix and the filler. ${ }^{[12]}$ When the temperature increases and exceeds a certain 


\section{WILEY-VCH}

threshold, the continuous network formed by the conductive fillers is disrupted. Due to expansion of the polymer matrix, the electrical conductivity and the current passing through the sample are reduced, regulating the temperature within a desired range. ${ }^{[13]}$ Great efforts have been made to design PTC materials which possess high PTC intensity (typically $>10^{3}-10^{4}$ ), good reproducibility of the PTC effect, sharp increase of resistivity over a narrow temperature range, tunable switching temperature and insensitivity to other external stimuli (such as strain, liquids, environment). ${ }^{[14]}$ Scientists have reported that a large number of factors can influence the PTC effect, including the choice of polymer matrix, the filler morphology and size and filler dispersion throughout the polymer matrix. ${ }^{[15]}$ Despite numerous investigations of the topic, a deterministic mechanism behind the pyro-resistive effect is still lacking, which makes the design and optimization of PTC devices difficult.

When designing a self-regulating heater, two equally important and fundamental aspects need to be considered: (i) large, sharp and repeatable PTC performance that self-regulates the heater; and (ii) efficient Joule heating to fulfil the heating purpose. ${ }^{[16]}$

Various conductive fillers and polymer matrices have been studied with the aim of obtaining a good PTC effect. Carbon blacks (CB) of different sizes have been studied by Luo and Wong, who claimed that $\mathrm{CB}$ with small surface area, large particle size and small amounts of aggregated structures lead to PTC behavior with a greater amplitude. ${ }^{[17]}$ Asare et al. also demonstrated that the PTC intensity increased with increasing filler size and decreasing filler content, for both spherical and flake-like "model" conductive fillers and mixed filler systems as larger fillers make less "robust" conductive filler networks. ${ }^{[10 a]}$ Therefore, there is a significant design compromise between good PTC behavior (for which relatively large filler size with low aspect ratio are desired) and good Joule heating properties (for which relatively high aspect ratio fillers are desired). 


\section{WILEY-VCH}

On the other hand, the selection of the polymer matrix in conductive composites plays also a crucial role. Semi-crystalline polymers such as high density polyethylene (HDPE) are often utilized in commercial self-regulating heating devices. Ultra-high molecular weight polyethylene (UHMWPE) and polyvinylidene fluoride (PVDF) have also been widely studied. ${ }^{[15 b, 18]}$ Although the toughness of these neat polymers is high, it drops dramatically with filler loading. In this work, thermoplastic elastomers are therefore selected for their high flexibility.

As mentioned earlier, a good level of Joule heating (also known as resistive heating) is essential in order to build up successful self-regulating heating devices. Carbon nanotube (CNT) filled polymer composites are ideal materials for practical heating elements due to their high electrical and thermal conductivity, high thermal and chemical stability, superior capability of carrying current, in combination with a low percolation threshold. ${ }^{[1 \mathrm{~b}, 19]}$ Unfortunately, their intrinsic negative temperature coefficient (NTC) effect (decrease of electrical resistance with temperature) has prevented so far any applications as self-regulating heaters. Raising temperature during pyro-resistive testing allows the CNTs within the polymer matrix to reagglomerate and establish particle-particle contacts, leading to so-called "dynamic percolation" and an increase in conductivity. ${ }^{[20]}$ To explore the possibility of obtaining both a low percolation threshold and high PTC intensity, direct mixing of two or more fillers has been attempted in the past. However, the results demonstrated that the PTC intensity of mixed filler systems is dominated by the low PTC intensity filler phase, even at very low volume fractions. ${ }^{[21]}$ Due to the complexity and sensitivity of the mixed filler system, a good switching heater cannot be obtained via this strategy. Very recently, an innovative composite system has been established to reach a zero temperature coefficient of resistance value using a bilayer structure, which consisted of a CNT-based layer (for the NTC effect) and a CB-based layer (for the PTC effect) to stabilize the electrical resistance change during Joule heating. ${ }^{[22]}$ Inspired by 


\section{WILEY-VCH}

these results, we aim here to create novel smart self-regulating heating devices with both high PTC intensity and efficient Joule heating, based on series and/or parallel connections of different CPCs. More generally we demonstrate a universal approach to provide unique control over pyro-resistive properties, by designing, testing and modelling a number of devices with different connecting configurations as well as different polymer matrices and conducting fillers.

\section{Results and Discussion}

\subsection{Morphology}

As an example of a series connected heating device, Figure 1a illustrates a device constructed with three components. CNT filled thermoplastic polyurethane (TPU) composites are located at both ends of the device and act as the heating elements while a silver coated glass sphere (AgS) of $50 \mu \mathrm{m}$ in diameter (Figure S1, see supporting information) filled TPU composite is located in the middle and acts as a self-regulating switch, owing to its large PTC intensity. ${ }^{[10 a]}$ A simple circuit diagram of the series connection based on the illustrated sample is shown in Figure 1a. The resistance can be represented by Equation 1 below, assuming that the contact resistance at the interface is ignored.

$R_{\text {series }}=R_{\text {heat }}+R_{\text {switch }}+R_{\text {heat }}$

Where $R_{\text {series }}$ is the overall resistance of the three-part series connected sample, $R_{\text {heat }}$ is the resistance of the heating component (i.e., the TPU/CNT composite) and $R_{\text {switch }}$ is the resistance of the PTC switching unit (i.e., the TPU/AgS composite).

The brittle cryogenic fracture surfaces of CNT and AgS loaded specimens were examined under a scanning electron microscope (SEM), as shown in Figure 1b-d. A relatively homogenous CNT dispersion is shown in Figure 1b, indicating that CNTs are locally well distributed within the TPU matrix. The good level of dispersion, especially at relatively high 


\section{WILEY-VCH}

CNT concentration (5 wt.\%) is ideal for Joule heating purposes. This good dispersion can be attributed to the high shear force applied during the twin-screw melt compounding process. ${ }^{[3 \mathrm{~b} \text {, }}$ ${ }^{23]}$ Figure 1d shows the SEM micrograph of specimen containing AgS (45 wt.\%) filler. These micro-sized fillers were distributed within the TPU matrix with better interfacial bonding and interaction between filler and polymer matrix, compared to HDPE/AgS composites, which showed obvious de-bonding as reported in our previous study. ${ }^{[10 a]}$ A clear and continuous joining line between TPU/CNTs and TPU/AgS segments was observed in Figure 1c, demonstrating good interfacial adhesion and contact.

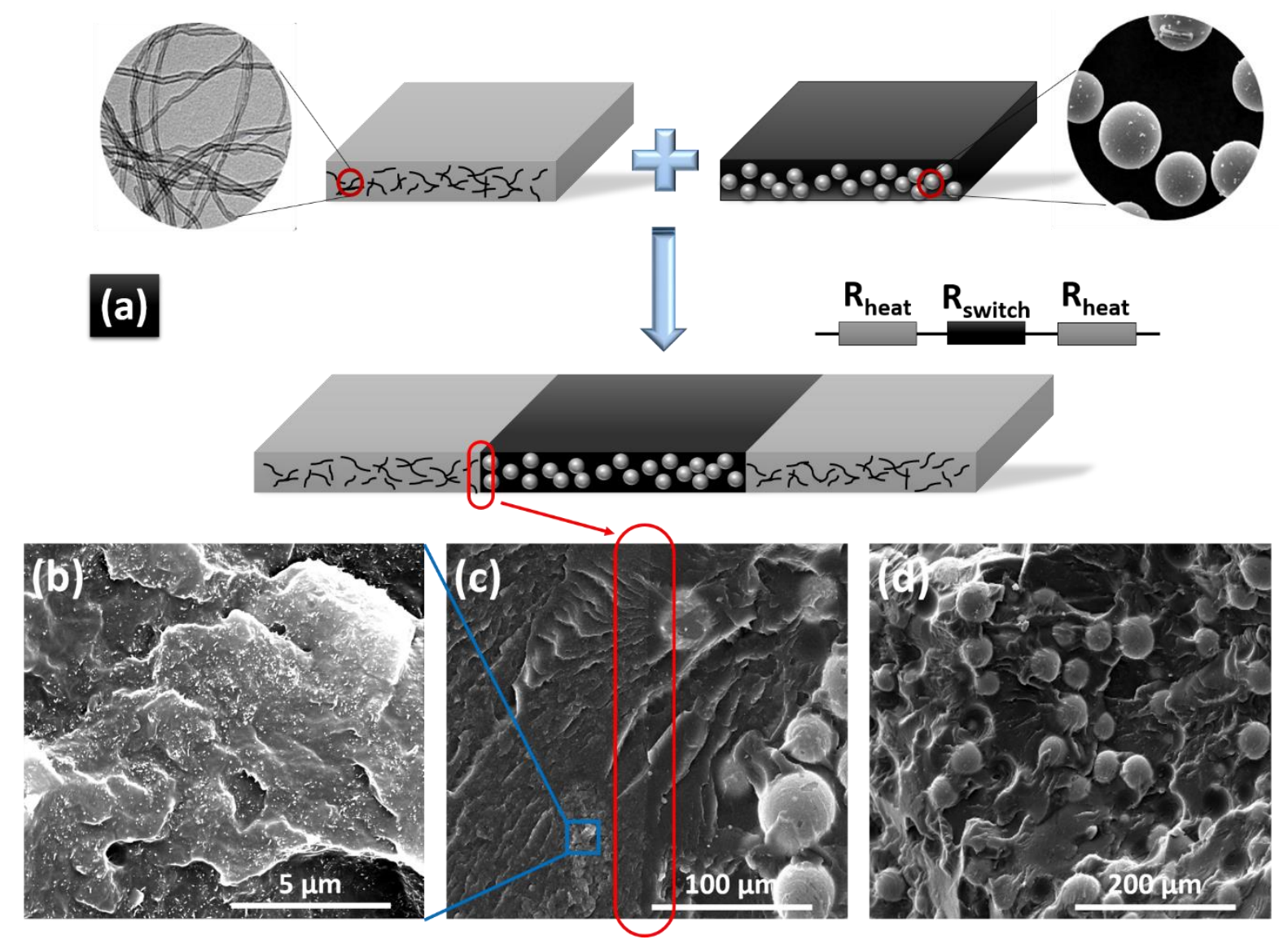

Figure 1. Schematic illustration and morphology of a tri-component series assembly device. a) An illustration of the self-regulating heating device consisting of a switching unit (TPU/AgS composite) in the middle and heating elements (TPU/CNT composite) at both ends; Scanning 
electron microscopy (SEM) images of b) TPU/CNT composite (5 wt.\%), c) interfacial regions between TPU/CNT and TPU/AgS segments and d) TPU/AgS composite (45 wt.\%).

\subsection{Statistical percolation of CNT and AgS in TPU}

Figure 2 shows the electrical conductivity of TPU composites containing CNT or AgS as a function of fillers content. Classical percolation theory is used to describe the conductivity change of TPU/CNT and TPU/AgS composites by a scaling law:

$\sigma=\sigma_{0}\left(\varphi-\varphi_{c}\right)^{t}$

Where $\sigma$ is the conductivity of $\mathrm{CPC}, \sigma_{0}$ is a scaling factor, $\varphi$ is the filler content and $\varphi_{c}$ is the percolation threshold, and $t$ is the critical exponent which is expected to depend on the conductive system dimensionality only. The percolation threshold can be determined by fitting the experimental data with Equation 2. ${ }^{[24]}$

For TPU/CNT samples (Figure 2a), the conductivity shows about a 4 orders of magnitude increase from $10^{-5} \mathrm{~S} / \mathrm{m}$ to $10^{-1} \mathrm{~S} / \mathrm{m}$ when the CNT loading increases from $0.3 \mathrm{wt} . \%$ to $1 \mathrm{wt} . \%$. Above $1 \mathrm{wt} . \%$, the conductivity reaches a plateau and only increases slightly with further loading. Fitting the data with Equation 2 gives a percolation threshold of 0.32 wt.\% (0.19 vol.\%), which is relatively low due to the dynamic percolated CNT network during compression molding. ${ }^{[3 \mathrm{~b}]}$ Unlike the exponential electrical conductivity change of TPU/CNT samples, the electrical conductivity change of TPU/AgS composites (Figure 2b) has a very sharp 'on-off' behavior. Above a certain AgS filler loading (40 wt.\% / 23.5 vol.\%), the conductivity is more or less constant, while below this loading, the samples are totally insulating. For this micro-sized spherical Ag coated filler system, the shape, size, and conductivity level of the silver coating are all affecting the percolation behavior of the composites, which is different from conductive nano-fillers. This is in accordance with studies 
by Grimaldi et al. who observed that a sharp cut-off applies well to CPCs made of large

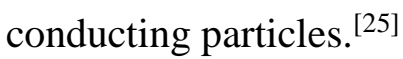
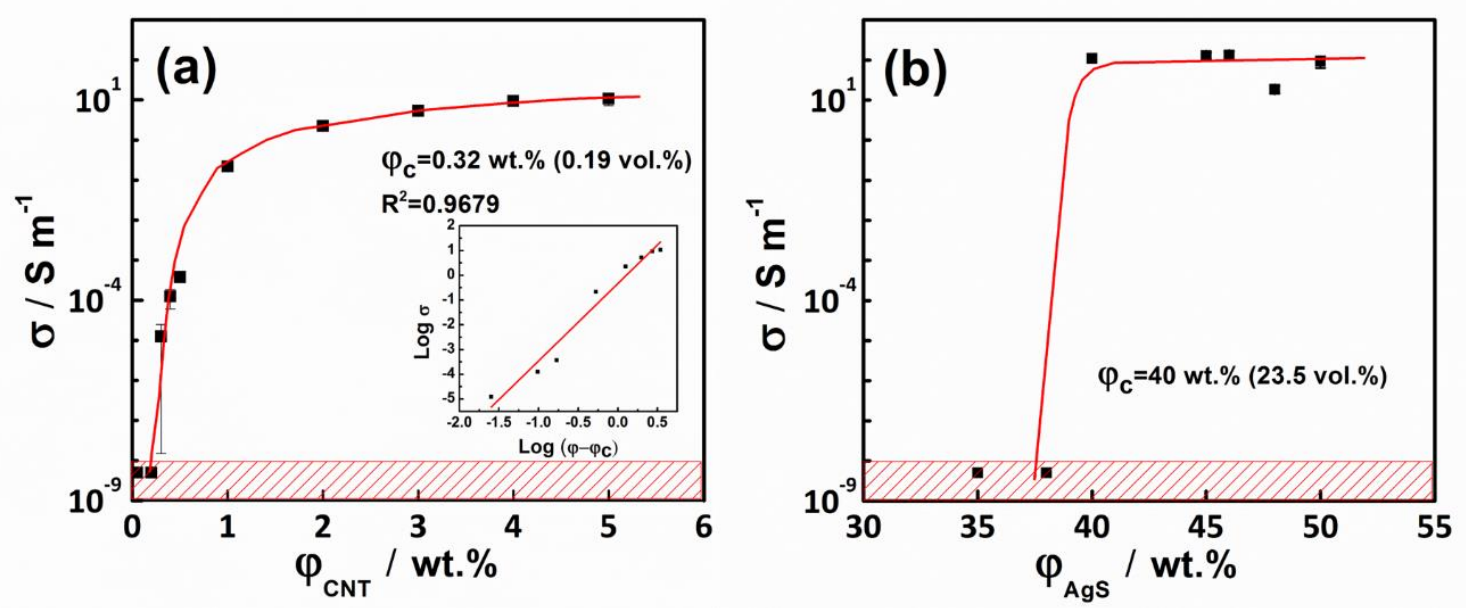

Figure 2. Percolation curves of a) TPU/CNT composites showing a relatively low percolation threshold $\left(\varphi_{c}\right)$ of $0.32 \mathrm{wt} . \%$, calculated by fitting the experimental data with Equation 2 (inset); and b) TPU/AgS composites showing a sharp "on-off" behavior in electrical conductivity in correspondence with the percolation threshold.

\subsection{Experimental and theoretical study of pyro-resistive behavior}

\subsubsection{Mono-filler systems}

To examine the pyro-resistive behavior of both TPU/CNT and TPU/AgS systems, the electrical resistivity of specimens was monitored as a function of temperature as shown in Figure 3a and b. With the aim of better understanding the pyro-resistive behavior at different filler loadings, two representative concentrations of both TPU/CNT composites ( 0.4 wt.\% and 5 wt.\%) and TPU/AgS composites (45 wt.\% and $50 \mathrm{wt} . \%$ ) were selected. The first concentration was close to, but above, the respective $\varphi_{c}$ while the second one was much higher than $\varphi_{c}$. 


\section{WILEY-VCH}

In Figure 3a, it can be observed that TPU/CNT composites with different concentrations $(0.4$ wt.\% and 5 wt.\%) showed a similar trend, where a gradual and steady decrease in resistivity upon heating (NTC effect) can be observed due to perfectioning of the CNT network after relaxation of polymer molecules (i.e. dynamic percolation). ${ }^{[26]}$ The opposite is observed for both TPU/AgS composites (45 wt.\% and 50 wt.\%) in Figure 3b, with a gradual increase in resistivity with increasing temperature followed by a sudden increase in resistivity (more than 6 orders of magnitude) at elevated temperature (PTC effect). This PTC effect from TPU/AgS specimens is an essential and fundamental feature for self-regulating purposes, therefore of particular interest for self-regulating heating devices, in order to avoid overheating. TPU/AgS composite (45 wt.\%), TPU/CNT composite (5 wt.\%) and TPU/CNT composite (0.4 wt.\%), are referred to as $R_{1}, R_{2}$ and $R_{3}$, respectively, and are connected in parallel and series as shown in the inset of Figure $3 c$ and $d$.

\subsubsection{Parallel and series connected systems}

With the aim of having a universal control on the self-regulating heating devices by utilizing the advantages of each system, both parallel and series connections between the two systems have been developed and characterized. In order to elucidate the mechanism, both theoretical predictions and experimental studies have been performed for both types of connection (Figure $3 \mathrm{c}$ and d). Considering the similar PTC behavior of TPU/AgS composite systems with $45 \mathrm{wt} \%$ and 50 wt.\% loadings, the lower filler loading (45 wt.\%) of the two was selected for the switching/self-regulating unit due to benefits in terms of costs, weight saving and the preservation of mechanical properties. Regarding the heating unit, two different TPU/CNT composites (0.4 wt.\% and $5 \mathrm{wt} . \%)$ were studied, due to the significant differences in their initial resistivity and to better analyse and understand the fundamental phenomenon of parallel and series connections. 


\section{WILEY-VCH}

In a parallel connected circuit design (Figure 3c), the current flow follows Kirchhoff's first law (current law). Therefore, as expected the currents flow through the pathways with the lowest electrical resistance. As a result, the overall resistivity of TPU/AgS (45 wt.\%) - TPU/CNT(5 wt.\%) parallel composites behave similarly to TPU/CNT (5 wt.\%) composite as indicated by the dashed line, without exhibiting any observable switching behavior. The TPU/AgS (45 wt.\%) - TPU/CNT (0.4 wt.\%) parallel composites on the other hand follow the same pyro-resistive behavior as the TPU/AgS (45 wt.\%) composite before switching, showing the same resistivity as the TPU/CNT (0.4 wt.\%) composite since the TPU/AgS (45 wt.\%) pathways have been "switched off" by the PTC effect at elevated temperature. As a result, it can be seen that the pyro-resistive behavior is dominated by the conductive unit with the lower PTC intensity for two composites of similar resistivity. However, by utilizing the unique feature of the presented parallel system, PTC intensity can be tailored to a desired value by adjusting the resistivity of the TPU/CNT composites. Therefore, by using different CNT loadings, a universal control of the final resistivity of the parallel system can be achieved.

Simple analytical theoretical models have also been developed for parallel connections following the basic rules of electrical circuits. The predicted parallel resistivity change shows a good agreement with experimental data for both CNT loadings, as is shown in Figure 3c (dashed lines). Model predictions for a parallel connection of TPU/AgS and TPU/CNT composites are based on experimental data of single filler-matrix systems as described in Equation 3 and Equation 4, where the total resistance of the parallel connected samples can be predicted as:

$$
\begin{aligned}
& R(T)_{\text {parallel (5wt.\%CNTs) }}=\left(\rho_{1}(T) \frac{\ell_{1}}{A_{1}} \times \rho_{2}(T) \frac{\ell_{2}}{A_{2}}\right) /\left(\rho_{1}(T) \frac{\ell_{1}}{A_{1}}+\rho_{2}(T) \frac{\ell_{2}}{A_{2}}\right) \\
& R(T)_{\text {parallel (0.4wt.\%CNTs) }}=\left(\rho_{1}(T) \frac{\ell_{1}}{A_{1}} \times \rho_{3}(T) \frac{\ell_{3}}{A_{3}}\right) /\left(\rho_{1}(T) \frac{\ell_{1}}{A_{1}}+\rho_{3}(T) \frac{\ell_{3}}{A_{3}}\right)
\end{aligned}
$$


Where $R(T)_{\text {parallel (5 wt.\%CNTs) }}$ and $R(T)_{\text {parallel }(0.4 w t . \% C N T s)}$ are the resistance of TPU/AgS (45 wt.\%) - TPU/CNT (5 wt.\%) parallel composites and TPU/AgS (45 wt.\%) - TPU/CNT (0.4 wt.\%) parallel composites depending on temperature, respectively. $\rho_{1}(T), \rho_{2}(T)$, and $\rho_{3}(T)$ are the resistivity of $R_{1}, R_{2}$ and $R_{3}$ depending on temperature, respectively. $\ell_{1}, \ell_{2}, \ell_{3}$ and $A_{1}, A_{2}, A_{3}$ are the length and cross-sectional area of $\mathrm{R}_{1}, \mathrm{R}_{2}$ and $\mathrm{R}_{3}$, respectively.

In the series system (Figure 3d), the overall pyro-resistive behavior and PTC intensity are very similar to that of the TPU/AgS composite, indicating the possibility of utilizing the series connected composites as a switch for heating devices. In other words, good pyro-resistive behavior dominated by the conductive composites with higher PTC intensity has been preserved using this series connection. Following Kirchhoff's second law (voltage law), the sum of all the voltages around this circuit loop is equal to zero. Thus, every component in the series system carries the same current. Interestingly, the initial resistivity of the device is skewed towards the composites with lower values of resistivity, closer to the value for TPU/CNT composites, as indicated by the dashed lines shown in Figure 3d. As a result, the series connected composites can benefit from both the switching unit of the TPU/AgS composite and the heating element of the TPU/CNT composite with a wide resistivity range to select specific Joule heating rates under the same applied voltage. A simple theoretical model has also been applied to the series system, where the overall resistivity is based on each of the single filler-matrix systems according to Equation 5 and Equation 6.

$$
\begin{aligned}
& R(T)_{\text {series }(5 w t . \% C N T s)}=\rho_{1}(T) \frac{\ell_{1}}{A_{1}}+\rho_{2}(T) \frac{\ell_{2}}{A_{2}} \\
& R(T)_{\text {series }(0.4 w t . \% C N T s)}=\rho_{1}(T) \frac{\ell_{1}}{A_{1}}+\rho_{3}(T) \frac{\ell_{3}}{A_{3}}
\end{aligned}
$$




\section{WILEY-VCH}

Where $R(T)_{\text {series }(5 w t . \% C N T s)}$ and $R(T)_{\text {series }(0.4 w t . \% C N T s)}$ are the resistance of TPU/AgS (45 wt.\%) - TPU/CNT (5 wt.\%) series composites and TPU/AgS (45 wt.\%) - TPU/CNT (0.4 wt.\%)

series composites depending on temperature. $\rho_{1}(T), \rho_{2}(T)$, and $\rho_{3}(T)$ are the resistivity of $\mathrm{R}_{1}, \mathrm{R}_{2}$ and $\mathrm{R}_{3}$ depending on temperature, respectively. $\ell_{1}, \ell_{2}, \ell_{3}$ and $A_{1}, A_{2}, A_{3}$ are the length and cross-sectional area of $\mathrm{R}_{1}, \mathrm{R}_{2}$ and $\mathrm{R}_{3}$, respectively.
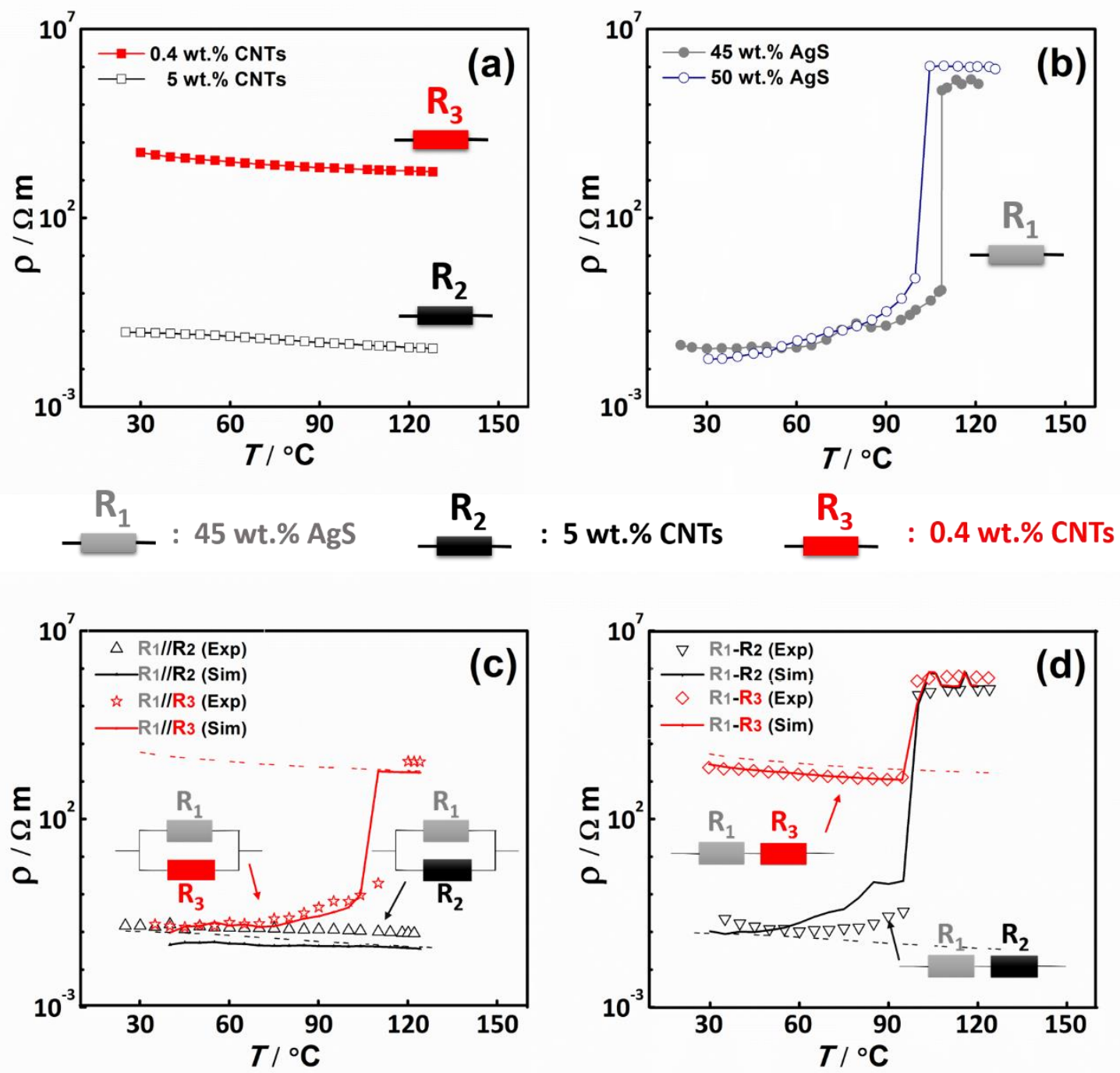

Figure 3. The pyro-resistive behavior of a) TPU/CNT composites (5 wt.\% and 0.4 wt.\%), showing a slight NTC effect at both loadings; b) TPU/AgS composites (45 wt.\% and 50 wt.\%), with a clear PTC effect at similar temperature; c) and d) predicted and experimentally measured electrical resistivity of TPU/AgS - TPU/CNT composites in parallel and series connection, 
respectively, as a function of temperature. TPU/AgS composite (45 wt.\%), TPU/CNT composite (5 wt.\%) and TPU/CNT composite (0.4 wt.\%) are referred to as R1, R2 and R3, respectively. (Dashed lines replicate the resistivity of TPU/CNT composites, 5 wt.\% and 0.4 wt.\% in $\mathrm{c}$ and $\mathrm{d}$, respectively). Excellent agreement between the experimental data and predicted pyro-resistive behavior has been obtained.

\subsection{Tri-component series assembly with different switching unit length}

To further investigate the series system after the experimental and theoretical study of the twopart series system, more practical designs were developed for self-regulating heating devices. In order to have a higher fraction of heating element and a more steady system, a tri-component series assembly was manufactured with heating elements (TPU/CNT composite (5 wt.\%)) on both ends and a switching unit (TPU/AgS composite (45 wt.\%)) in the middle as shown in Figure 4 inset illustration. A switching behavior similar to the TPU/AgS (45 wt.\%) system was observed with three representative switching unit length ratios in the middle $(2: 1: 2,1: 1: 1$, and 1:2:1) as shown in Figure 4a, confirming the effectiveness of the series system which combines and exhibits both the switching and heating functions at various different switching unit proportions. These results demonstrate that the fraction of the switching unit (TPU/AgS composite) in the series composites does not affect the switching behavior of the series system within a certain range. Although all the systems studied have been proven fully functional for self-regulating purposes, independently from the switching/heating unit ratios, it is worth noting that, from a practical design point of view, the switching unit length and thickness needs to be larger than a lower limit in order to avoid short circuits and to guarantee sufficient matrix expansion upon heating.

It is worth noting that a zero temperature coefficient has been realized before the PTC transition in the tri-component series assembly, which is essential for the precise control of the 


\section{WILEY-VCH}

temperature in heating applications. Compared to the previously reported TPU/AgS composite (45 wt.\%) and two-part series composite systems, the tri-component series design can establish a more reliable heating system for practical heating devices. Apart from a good level of Joule heating introduced by the TPU/CNT composites within tri-component series assembled systems, the other obvious benefit from employing CNTs over micron-size low aspect ratio fillers (such as $\mathrm{AgS}$ ) is the cost and weight saving for such a system.

The repeatability and stability of the heating units are important factors in determining the quality of the heating devices. Three repeated heating cycles were carried out on tri-component series assembled samples with the smallest switching unit portion $(2: 1: 2)$ as shown in Figure $4 \mathrm{~b}$, confirming repeatability and stability of the device. The repeatability of different switching unit portions was also been confirmed with 3 cycles of external heating (Figure S2, supporting information).
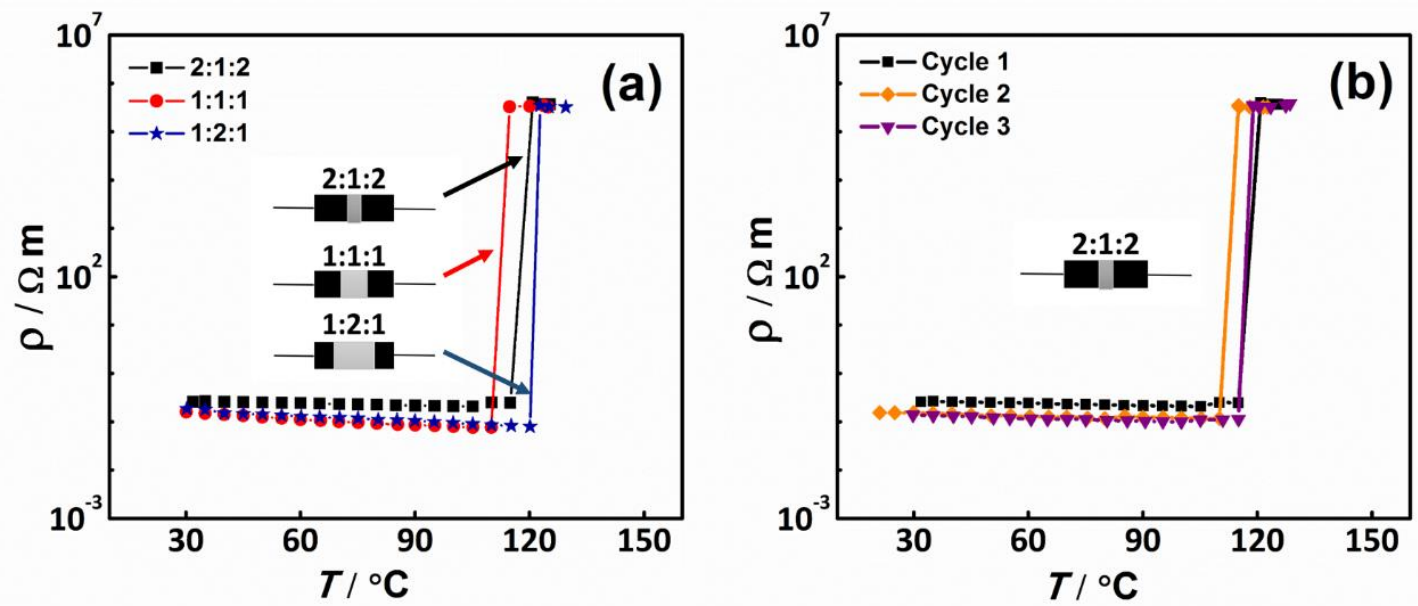

Figure 4. The pyro-resistive behavior of tri-component series assembly devices. a) with three representative switching unit length ratios (TPU/AgS composites) in the mid-section $(2: 1: 2$, $1: 1: 1$, and 1:2:1); and b) three repeated heating cycles on the tri-component series sample with the smallest switching unit portion $(2: 1: 2)$, showing good repeatability of the presented systems. 


\section{WILEY-VCH}

\subsection{Joule heating behavior}

As mentioned earlier, an efficient Joule heating property is essential for a good heating device, regardless of their self-regulating functionality. Although TPU/AgS composites showed a large PTC intensity, the heating temperature they can ultimately reach is rather limited. ${ }^{[22]}$ In Figure $5 \mathrm{a}$ and $\mathrm{b}$, it can be observed that by applying $5 \mathrm{~V}$, the overall temperature can only reach around $60{ }^{\circ} \mathrm{C}$ for TPU/AgS composites, while the TPU/CNT composite heats up more rapidly and reaches a temperature of more than $180^{\circ} \mathrm{C}$ (Figure $\left.5 \mathrm{c}-\mathrm{d}\right)$ with the trend of over-heating.

Joule heating of the tri-component series assembly is presented in Figure 5e-j. The universality of the approach allows the composites to achieve series connections either in a line or sandwich structure. In this case, the change in temperature was recorded under an applied voltage of $5 \mathrm{~V}$ to the sandwich tri-component series assembly structure as illustrated in Figure 5e, with TPU/CNT composites (5 wt.\%) as top and bottom layers and TPU/AgS composite (45 wt.\%) positioned in the middle. When the voltage was applied to the specimen, it started to heat up within a relatively short period of time and then saturated and stabilized to the PTC switching temperature $\left(\sim 110^{\circ} \mathrm{C}\right)$ of the switching unit as shown in Figure 5f. Good temperature uniformity was confirmed by thermal infrared (IR) camera images shown in Figure 5g.

To further explore and demonstrate the universality and versatility of this approach, different polymers and fillers have been used for both the switching unit and the heating unit. For instance, HDPE is used instead of TPU in the switching unit to achieve a higher switching temperature (expected at $\sim 130{ }^{\circ} \mathrm{C}$ ). ${ }^{[27]}$ Graphene nanoplatelets (GNPs) were also used instead of AgS. Linear tri-component series samples of HDPE/GNP (24 wt.\%) and HDPE/CNT (5 wt.\%) have been manufactured (Figure 5h), showing a heating and temperature regulating behavior similar to the series samples of TPU/AgS (45 wt.\%) and TPU/CNT (5 wt.\%). The temperature change with time was demonstrated for HDPE/GNP (24 wt.\%) and HDPE/CNT 


\section{WILEY-VCH}

(5 wt.\%) series systems by applying a voltage of $240 \mathrm{~V}$, with two switching unit length ratios

(1:1:1 and $1: 2: 1)$. A temperature plateau at around $120{ }^{\circ} \mathrm{C}$ was reached for both composites which is slightly below the melting temperature of the HDPE matrix as shown in Figure 5i. From these two curves, it can be observed that, with a larger portion of heating units (HDPE/CNT composites), the time required to reach the equilibrium temperature was reduced. In other words, heating is faster in devices with larger heating units (Figure S3, supporting information). The IR image in Figure 5j also shows a uniform temperature distribution.

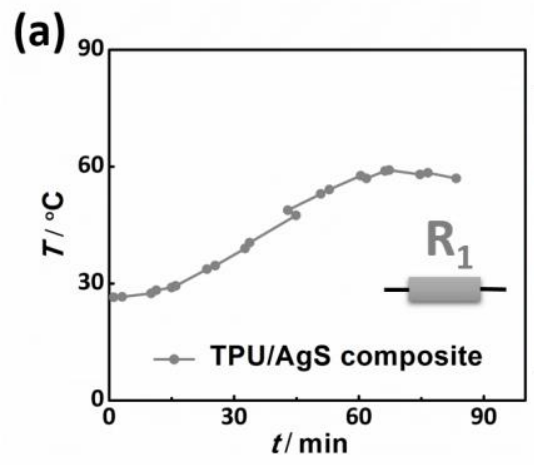

(e)

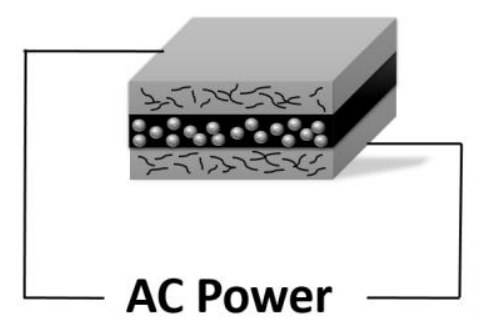

(h)

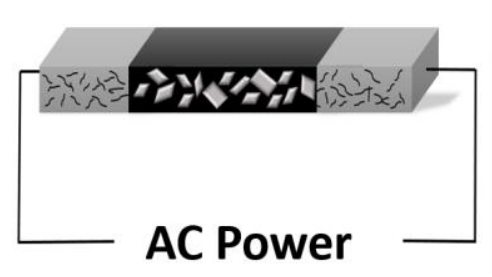

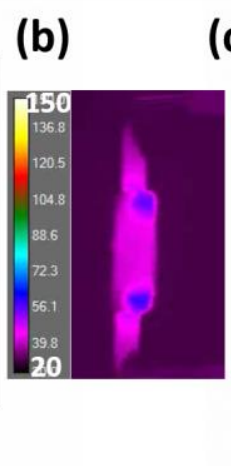

(f)

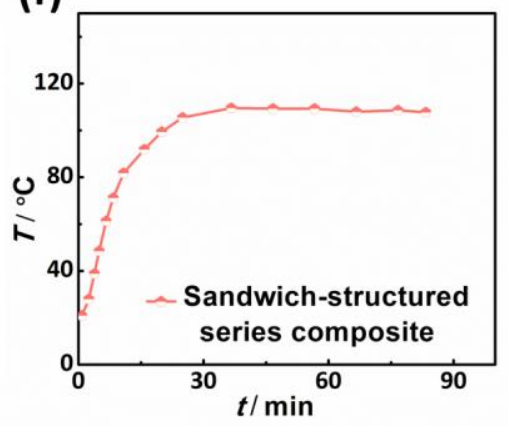

(i)

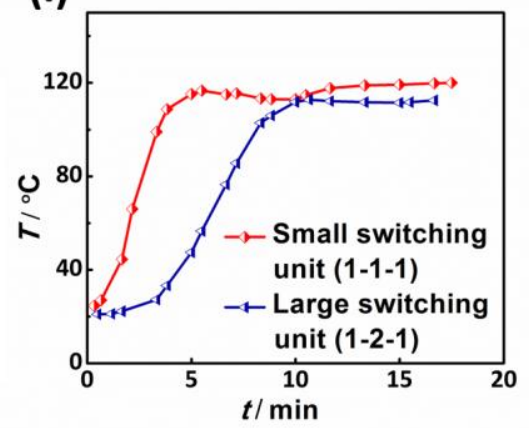

(c)

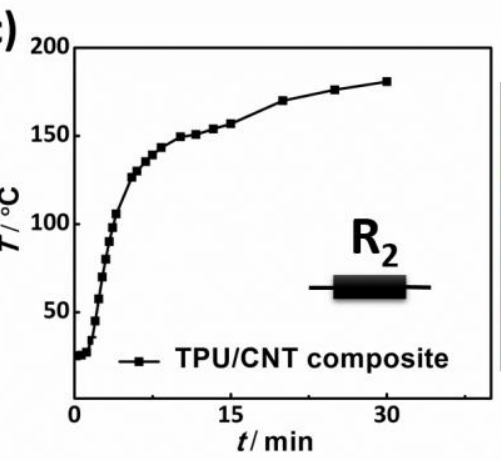

(d)

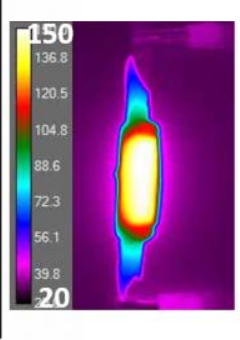

(g)

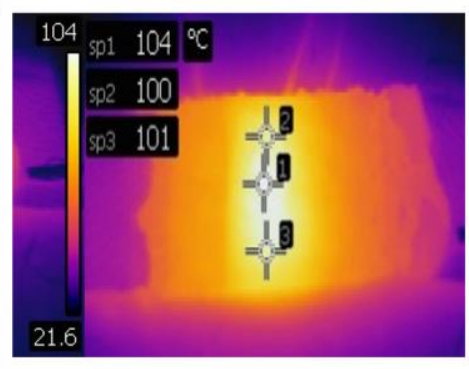

(j)

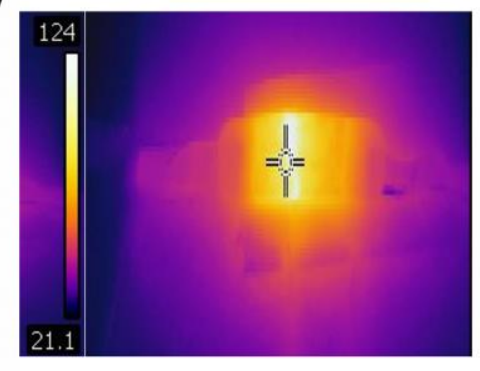

Figure 5. Device illustration, Joule heating behavior, and IR images of: TPU/AgS composite reaching $60{ }^{\circ} \mathrm{C}$ after $60 \mathrm{~min}\left(\mathrm{a}\right.$ and b); TPU/CNT composite reaching more than $150{ }^{\circ} \mathrm{C}$ after 


\section{WILEY-VCH}

15 min (c and d); Sandwich based tri-component series assembly device with TPU/AgS as the switching unit which stabilized at $110^{\circ} \mathrm{C}$ (e, f and g); Linear tri-component series assembly device with HDPE/GNP as the switching part, showing universality of the current design with two switching unit length ratios $(1: 1: 1$ and $1: 2: 1)(h, i$ and $j)$. Uniform heating of the samples via Joule heating was confirmed by the IR images.

\subsection{Flexibility and robustness}

For many heating devices such as wearable smart heaters and heating mats, good mechanical flexibility and robustness is an important requirement. Unfortunately, polymers used in typical self-regulating compounds (e.g. HDPE), particularly with a high filler content and high crosslink density, show often a rather brittle mechanical behavior. The use in this case of TPU as the polymer matrix, combined with the series configuration and the fillers selected, significantly improved the flexibility of such a heating device. Flexibility is examined by imposing different radii of curvatures on the device, whilst measuring variations in electrical resistance (Figure 6a).

No obvious changes in electrical resistance were observed for any of the radii of curvatures tested (Figure 6b), suggesting that there was no severe break-down of conductive pathways inside the specimen upon bending. Moreover, heating is also not affected by bending as demonstrated in Figure $6 \mathrm{c}$ and d. 
(a)

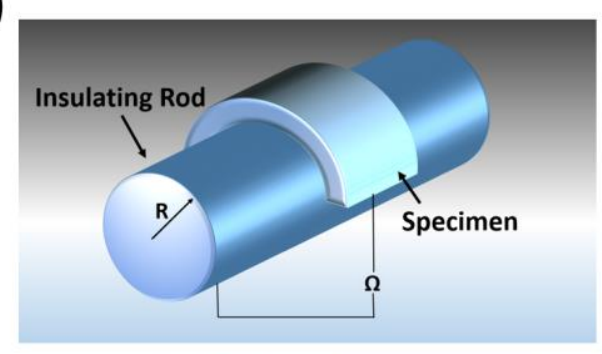

(c)

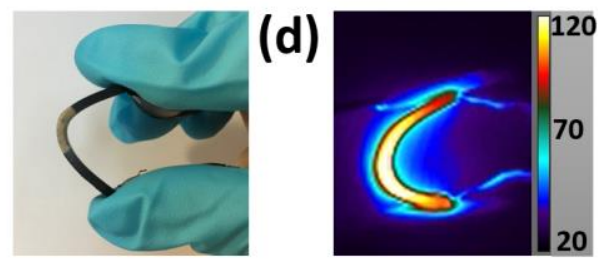

(b)

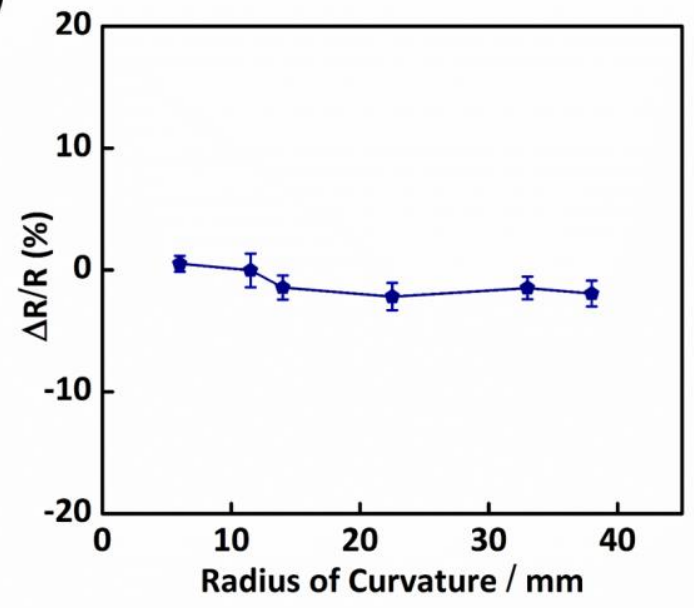

Figure 6. a) Illustration of the tri-component series assembly based on TPU matrix, b) relative resistance change of tri-component series assembly upon bending at different radius of curvatures, confirming the good flexibility, robustness and reliability of the presented devices. Both c) and d) demonstrate the flexibility of the specimen and the IR image under Joule heating.

\section{Conclusions}

In this work, a simple and versatile approach is presented that tackles typical design compromises in smart self-regulating heating devices by connecting different CPCs in series with distinct pyro-resistive behaviors. The universality of this approach was demonstrated both experimentally and theoretically by testing and modelling a number of devices with different connection configurations (in series or parallel) as well as different polymer matrices and conducting fillers. A tri-component series connected heating device has been successfully developed and characterized. This device shows for the first time high PTC intensity combined with good Joule heating as well as mechanical flexibility and robustness. Experimental results from both external heating tests and self-heating tests revealed that the pyro-resistive behavior was dominated by the conductive composites with high PTC intensity in the series connection, while all devices showed self-regulating behavior at their PTC temperature. As a result, 
switching function/requirement can be fulfilled in a universal manner by assembling CPCs with targeted PTC and Joule heating behavior.

\section{Experimental Section}

\section{Materials}

All the polymers are in the form of pellets and dried overnight at $80{ }^{\circ} \mathrm{C}$ before compounding. Details of materials are listed in Table 1.

Table 1. Polymer and conductive filler information.

\begin{tabular}{|c|c|c|c|}
\hline & MATERIAL & TRADE NAME & INFORMATION \\
\hline \multirow{2}{*}{ POLYMER } & Thermoplastic polyurethane (TPU) & Lubrizol Estane ${ }^{\circledR} 58437$ & density $1.19 \mathrm{~g} \mathrm{~cm}^{-1}$ \\
\hline & High density polyethylene (HDPE) & Rigidex® HD5218EA & density $0.952 \mathrm{~g} \mathrm{~cm}^{-1}$ \\
\hline \multirow{3}{*}{$\begin{array}{l}\text { CONDUCTIVE } \\
\text { FILLER }\end{array}$} & Silver coated glass spheres (AgS) & Potters Industries Ltd. & average diameter $50 \mu \mathrm{m}$ \\
\hline & $\begin{array}{l}\text { Multi-wall carbon nanotubes } \\
\text { (MWCNTs) }\end{array}$ & $\begin{array}{l}\text { Nanocyl S.A. Product No. } \\
\text { C7000 }\end{array}$ & $\begin{array}{l}\text { average diameter } 9.5 \mathrm{~nm} \text {, carbon } \\
\text { purity } 90 \%\end{array}$ \\
\hline & Graphene nanoplatelets (GNPs) & $x G n P^{\circledR}$ Grade $M$ & $\begin{array}{l}\text { average diameter } 15 \mu \mathrm{m} \text {, density } \\
2.2 \mathrm{~g} \mathrm{~cm}^{-1} \text {, carbon content }>99.5 \%\end{array}$ \\
\hline
\end{tabular}

\section{Sample preparation}

In order to avoid damage to the silver coating on $\mathrm{AgS}$ particles during compounding, a corotating DSM X'plore MC 15 micro-compounder (Netherlands) was used to produce the TPU/AgS compounds, with a modest rotating speed at $50 \mathrm{rpm}$ for $5 \mathrm{~min}$, at a temperature of $200{ }^{\circ} \mathrm{C}$ under nitrogen atmosphere. CNTs (5 wt.\%) were compounded with TPU using a Dr. Collin ZK 25 twin-screw compounder (Germany) with a screw length of $40 \mathrm{D}$. The throughput was of $2 \mathrm{~kg} / \mathrm{h}$, using a screw speed of $50 \mathrm{rpm}$, and a temperature range between $190{ }^{\circ} \mathrm{C}$ and $220{ }^{\circ} \mathrm{C}$, over 8 heating zones. The produced TPU/CNT (5 wt.\%) compound was used as a master batch that was diluted into desired concentrations from $0.1 \mathrm{wt} . \%$ to $4 \mathrm{wt} . \%$ using the DSM X'plore MC 15 mini-compounder with the same mild processing conditions as used for 


\section{WILEY-VCH}

TPU/AgS composites. HDPE/GNP (24 wt.\%) composites were compounded in the Dr. Collin ZK 25 twin-screw compounder using a screw speed of $50 \mathrm{rpm}$ and a temperature range between $180{ }^{\circ} \mathrm{C}$ and $220{ }^{\circ} \mathrm{C}$, over 8 heating zones. The extruded strands that were produced were then chopped into pellets and compression molded into rectangular shaped samples with dimensions of $30 \mathrm{~mm} \times 10 \mathrm{~mm} \times 2 \mathrm{~mm}$, using a Dr. Collin hot press P300E, at $220{ }^{\circ} \mathrm{C}$ for $5 \mathrm{~min}$ and 60 bar pressure. Two pieces of copper mesh $(0.263 \mathrm{~mm}$ aperture and $0.16 \mathrm{~mm}$ wire diameter $)$ were embedded on both sides of the sample during compression molding for use as the electrodes. The series and parallel samples were manufactured by cutting the desired lengths of each composite, and hot welded the cut composite sections together using the same compression molding equipment.

\section{Characterization}

A scanning electron microscope (SEM) (FEI Inspector-F, Netherlands) was used to examine the morphology of sample cross-sections as well as the interfacial area between the TPU/CNT and TPU/AgS, with the aim to characterize the filler-filler, filler-polymer and compositecomposite interaction. Brittle fracture was induced by immersing the specimens into liquid nitrogen for $5 \mathrm{~min}$. All analyzed surfaces were gold sputtered before imaging.

The pyro-resistive behavior of all samples was tested with an apparatus consisting of a temperature controlled oven (heating rate of $2{ }^{\circ} \mathrm{C} / \mathrm{min}$ ) and a two-point resistance measurement unit, obtained by combining a picoammeter (Keithley 6485) with a DC voltage source (Agilent 6614C). The thermocouple was placed close to, but not in contact with the specimen to ensure accurate reading. A constant voltage $(1 \mathrm{~V})$ was applied during heating and cooling cycles on the rectangular specimens while current and temperature were monitored and recorded simultaneously. 


\section{WILEY-VCH}

To evaluate the Joule heating behavior of the series composites, direct voltage was applied to the sample whilst two thermal infrared (IR) cameras (FLIR A35 and E40) recorded thermal images during heating.

To examine the flexibility and robustness of the produced specimens, the electrical resistance was measured in-situ during bending tests on the tri-component series assembly. Specimens were bent around an insulating cylindrical object of known radius while the electrical resistance was measured and recorded.

\section{Supporting information}

Supporting information is available from Wiley Online Library or from the author.

\section{Acknowledgement}

The authors gratefully acknowledge financial support from LMK Thermosafe Ltd., and also appreciate the technical assistant from Miss Yinping Tao from the Soft Matter group at QMUL.

\section{Reference}

[1] a) H. Deng, L. Lin, M. Ji, S. Zhang, M. Yang, Q. Fu, Progress in Polymer Science 2014, 39, 627; b) J. N. Coleman, U. Khan, W. J. Blau, Y. K. Gun'ko, Carbon 2006, 44, 1624; c) W. Zhang, A. A. Dehghani-Sanij, R. S. Blackburn, Journal of Materials Science 2007, 42, 3408.

a) H. Zhang, E. Bilotti, T. Peijs, Nanocomposites 2015, 1, 167; b) H. Zhang, Y. Liu, M. Kuwata, E. Bilotti, T. Peijs, Composites Part A: Applied Science and Manufacturing 2015, 70, 102; c) O. T. Picot, V. G. Rocha, C. Ferraro, N. Ni, E. D'elia, S. Meille, J. Chevalier, T. Saunders, T. Peijs, M. J. Reece, Nature Communications 2017, 8,14425; d) H. Zhang, Y. Liu, E. Bilotti, T. Peijs, Adv. Compos. Lett 2015, 24, 92. 


\section{WILEY-VCH}

[3] a) R. Zhang, H. Deng, R. Valenca, J. Jin, Q. Fu, E. Bilotti, T. Peijs, Composites Science and Technology 2013, 74, 1; b) E. Bilotti, R. Zhang, H. Deng, M. Baxendale, T. Peijs, Journal of Materials Chemistry 2010, 20, 9449; c) M. K. Choi, I. Park, D. C. Kim, E. Joh, O. K. Park, J. Kim, M. Kim, C. Choi, J. Yang, K. W. Cho, Advanced Functional Materials 2015, 25, 7109; d) R. Zhang, M. Baxendale, T. Peijs, Physical Review B 2007, 76, 195433.

[4] T. Villmow, S. Pegel, A. John, R. Rentenberger, P. Pötschke, Materials Today 2011, 14,340 .

[5] a) J. Lu, B. Kumar, M. Castro, J.-F. Feller, Sensors and Actuators B: Chemical 2009, 140, 451; b) J. F. Feller, Y. Grohens, Synthetic Metals 2005, 154, 193.

[6] F. Mai, Y. Habibi, J.-M. Raquez, P. Dubois, J.-F. Feller, T. Peijs, E. Bilotti, Polymer $2013,54,6818$.

[7] A. Rybak, G. Boiteux, F. Melis, G. Seytre, Composites Science and Technology 2010, $70,410$.

[8] a) X. Chen, T. Jiang, Y. Yao, L. Xu, Z. Zhao, Z. L. Wang, Advanced Functional Materials 2016, 26, 4906; b) F. Carpi, G. Frediani, S. Turco, D. De Rossi, Advanced Functional Materials 2011, 21, 4152.

[9] E. Smela, Advanced Materials 2003, 15, 481.

[10] a) E. Asare, J. Evans, M. Newton, T. Peijs, E. Bilotti, Materials \& Design 2016, 97 , 459; b) Z. Chen, P.-C. Hsu, J. Lopez, Y. Li, J. W. F. To, N. Liu, C. Wang, Sean C. Andrews, J. Liu, Y. Cui, Z. Bao, Nature Energy 2016, 1, 15009.

[11] C. Zhang, C.-A. Ma, P. Wang, M. Sumita, Carbon 2005, 43, 2544.

[12] K. Matsushige, K. Kobayashi, N. Iwami, T. Horiuchi, E. Shitamori, M. Itoi, Thin Solid Films 1996, 273, 128.

[13] K. Ohe, Y. Naito, Japanese Journal of Applied Physics 1971, 10, 99.

[14] H. Xu, Y. Wu, D. Yang, J. Wang, H. Xie, Rev. Adv. Mater. Sci 2011, 27, 173. 


\section{WILEY-VCH}

[15] a) H. Zhou, Z. Xia, X. Wang, Z. Li, T. Li, F. Guo, Polymer Composites 2015, 36, 1371;

b) J.-W. Zha, W.-K. Li, R.-J. Liao, J. Bai, Z.-M. Dang, J. Mater. Chem. A 2013, 1, 843.

[16] R. Menzel, S. Barg, M. Miranda, D. B. Anthony, S. M. Bawaked, M. Mokhtar, S. A.

Al- Thabaiti, S. N. Basahel, E. Saiz, M. S. Shaffer, Advanced Functional Materials 2015, 25, 28.

[17] S. Luo, C. Wong, IEEE Transactions on Components and Packaging Technologies $2000,23,151$.

[18] a) Y. Zeng, G. Lu, H. Wang, J. Du, Z. Ying, C. Liu, Scientific reports 2014, 4,6684; b) A. Kono, K. Shimizu, H. Nakano, Y. Goto, Y. Kobayashi, T. Ougizawa, H. Horibe, Polymer $2012,53,1760$.

[19] a) Y. H. Yoon, J. W. Song, D. Kim, J. Kim, J. K. Park, S. K. Oh, C. S. Han, Advanced Materials 2007, 19, 4284; b) K.-Y. Chun, Y. Oh, J. Rho, J.-H. Ahn, Y.-J. Kim, H. R. Choi, S. Baik, Nature nanotechnology 2010, 5, 853; c) K. Chu, D.-J. Yun, D. Kim, H. Park, S.-H. Park, Organic Electronics 2014, 15, 2734; d) K. H. Baloch, N. Voskanian, M. Bronsgeest, J. Cumings, Nature Nanotechnology 2012, 7, 316.

[20] H. Zhang, E. Bilotti, W. Tu, C. Y. Lew, T. Peijs, European Polymer Journal 2015, 68, 128.

[21] E. Asare, A. Basir, W. Tu, H. Porwal, H. Zhang, Y. Liu, J. Evans, M. Newton, T. Peijs, E. Bilotti, Nanocomposites 2016, 2, 58.

[22] K. Chu, S. C. Lee, S. Lee, D. Kim, C. Moon, S. H. Park, Nanoscale 2015, 7, 471.

[23] T. Villmow, P. Pötschke, S. Pegel, L. Häussler, B. Kretzschmar, Polymer 2008, 49, 3500.

[24] W. Bauhofer, J. Z. Kovacs, Composites Science and Technology 2009, 69, 1486.

[25] a) G. Ambrosetti, I. Balberg, C. Grimaldi, Physical Review B 2010, 82, 134201; b) I. Balberg, Journal of Physics D: Applied Physics 2009, 42, 064003. 


\section{WILEY-VCH}

[26] E. Bilotti, H. Zhang, H. Deng, R. Zhang, Q. Fu, T. Peijs, Composites Science and Technology 2013, 74, 85 .

[27] Y. Luo, G. Wang, B. Zhang, Z. Zhang, European polymer journal 1998, 34, 1221. 


\section{WILEY-VCH}

\section{The table of contents}

Flexible self-regulating heating devices, using a simple and universal materials design strategy, provide a unique control over pyro-resistive behavior by connecting different CPCs in series. A tri-component series assembled heating device has been successfully developed and characterized. This device combines for the first time high PTC intensity with good Joule heating as well as mechanical flexibility and robustness.

\section{Keywords}

Conductive polymer composites, positive temperature coefficient, smart heaters, selfregulating, carbon nanotubes

Y. Liu, H. Zhang, W. Tu, H. Porwal, J. Evans, M. Newton, J.J.C. Busfield, T. Peijs, E. Bilotti*

\section{Universal Control on Pyro-resistive Behavior of Flexible Self-regulating Heating} Devices

ToC figure

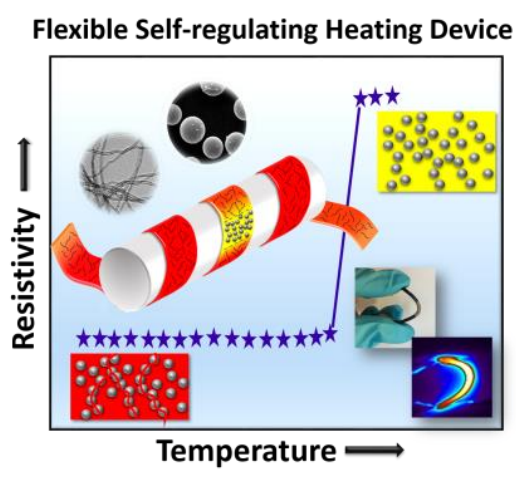

Copyright WILEY-VCH Verlag GmbH \& Co. KGaA, 69469 Weinheim, Germany, 2016. 


\section{Supporting Information}

\section{Universal Control on Pyro-resistive Behavior of Flexible Self-regulating Heating Devices}

Yi Liu, Han Zhang, Wei Tu, Harshit Porwal, Jamie Evans, Mark Newton, James Busfield, Ton Peijs, and Emiliano Bilotti*
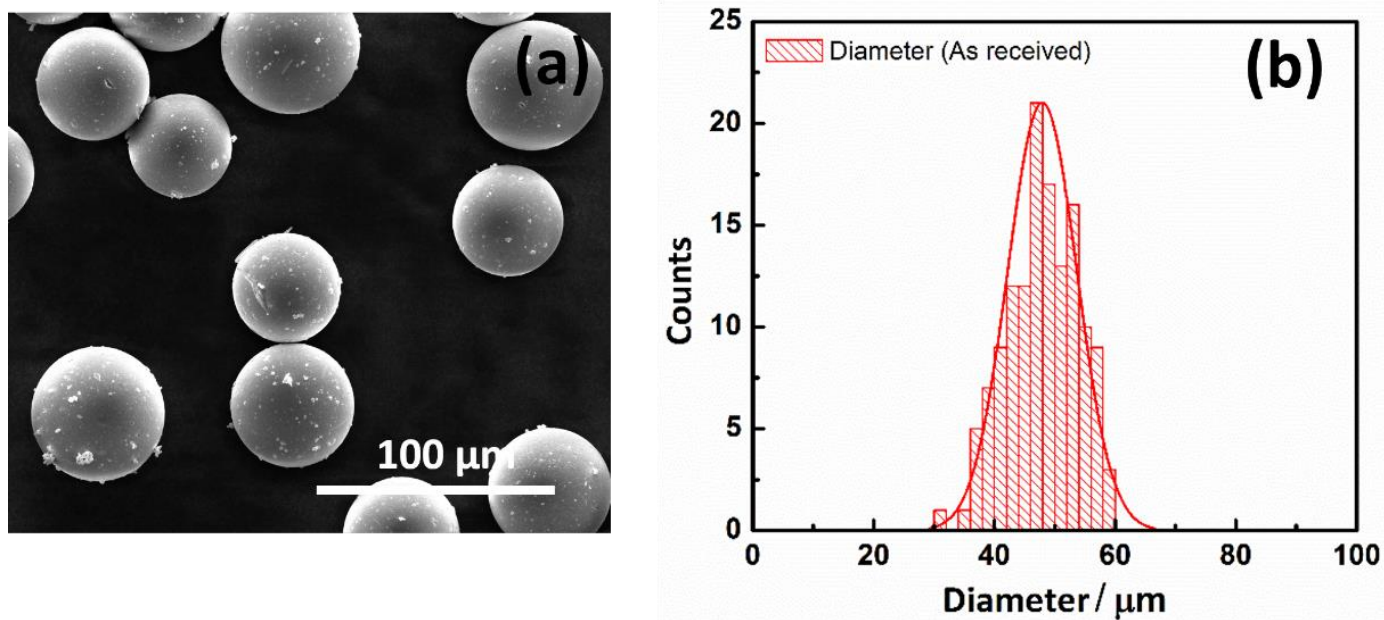

Figure S1. a) SEM images of silver coated glass spheres, b) Particle size distribution of asreceived silver coated glass spheres. The particle size distribution is relatively narrow, with most of the particle diameters between $30 \mu \mathrm{m}$ and $60 \mu \mathrm{m}$. The average filler diameter measured is $47.9 \mu \mathrm{m}$, in agreement with data sheet values from manufacturer. 
WILEY-VCH
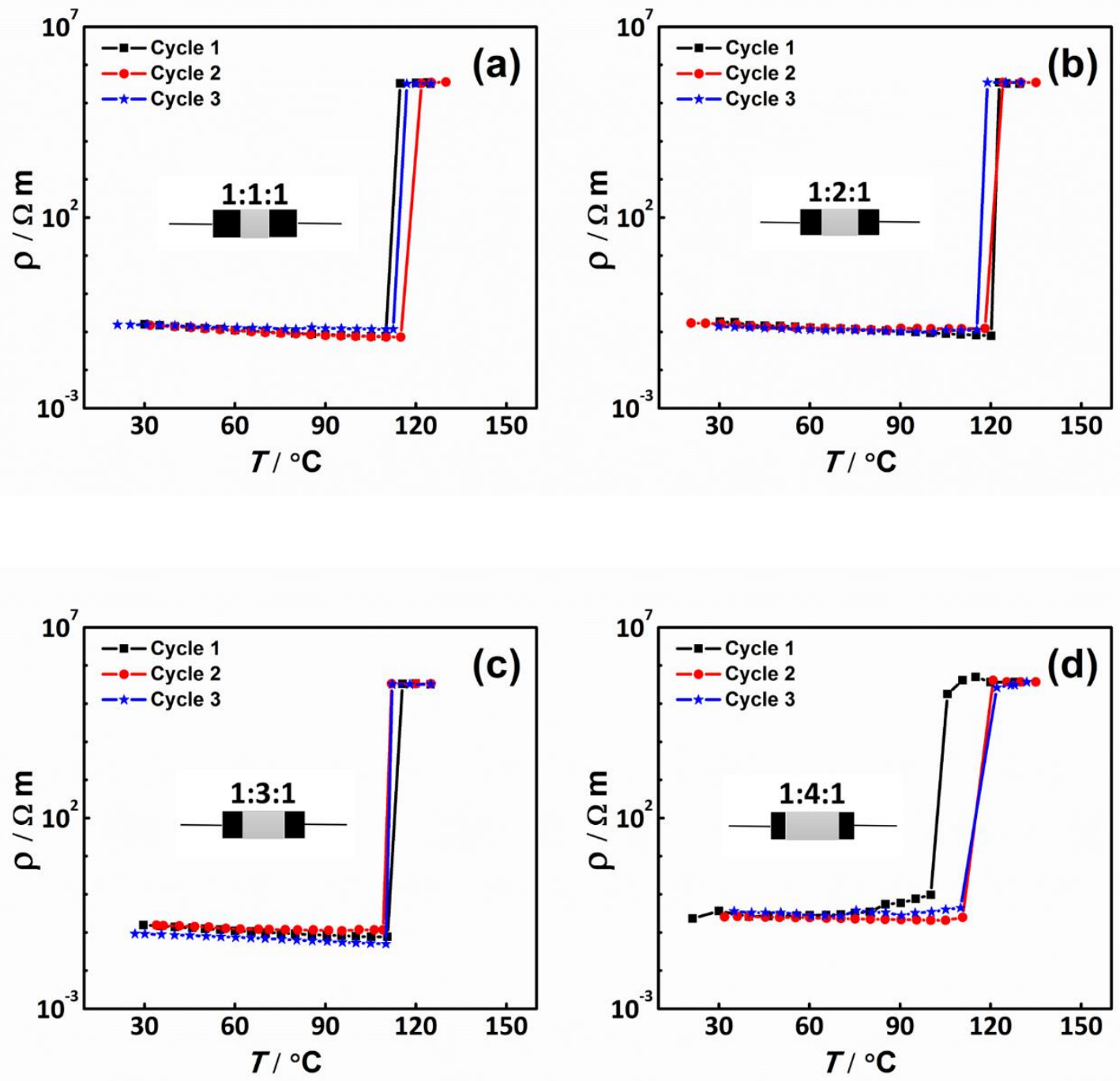

Figure S2. Pyro-resistive behaviors of tri-component series assembled samples with four different switching unit portions $(1: 1: 1,1: 2: 1,1: 3: 1$, and 1:4:1). Three repeated heating cycles were performed, confirming the repeatability and stability of the devices, independently of switching unit size. 

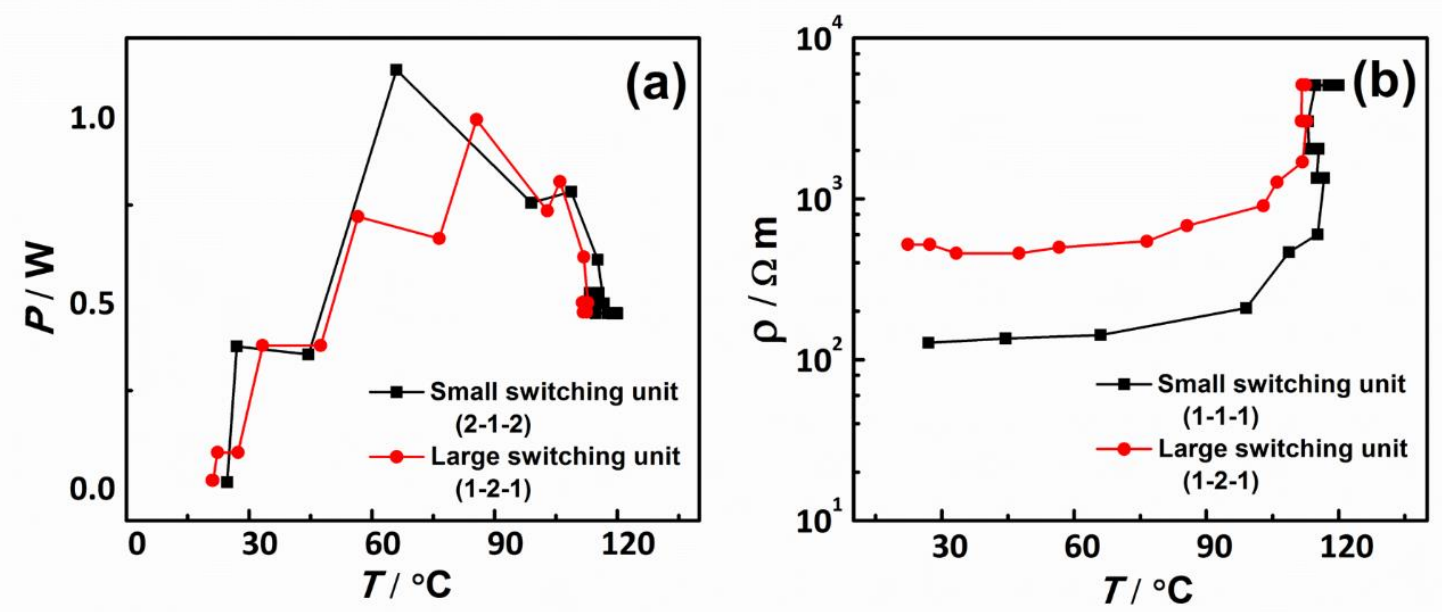

Figure S3. Joule heating performance of the tri-component series assembly with different ratios of HDPE/GNP composite as the switching part: a) Electrical power changes with increasing temperature and stabilized at the PTC switching temperature, indicating that no further heating up will occur. b) Resistivity increases with electrical heating for both assemblies and shows a stable final resistivity at the self-regulating temperature. Initial resistivity differences are observed for the two switching unit ratios, indicating different heating up rates. 